\title{
Differential avian and human tuberculin skin testing in non-tuberculous mycobacterial infection
}

\author{
A J Daley, D Isaacs
}

\begin{abstract}
Objective-To determine the sensitivity of differential avian and human delayedtype hypersensitivity skin testing in the diagnosis of non-tuberculous mycobacterial lymphadenitis.

Method-Retrospective review of all patients with culture proved nontuberculous mycobacterial lymph node infections who also had differential avian and human skin testing performed over a 10 year period from 1986 to 1996 .

Results-One hundred and twenty four patients had non-tuberculous mycobacteria isolated from lymph nodes over this period, 59 of whom had differential skin testing performed. The sensitivity of a response of $\geqslant 10 \mathrm{~mm}$ to the avian precipitin was 58 of 59. No patient had both a negative human and avian Mantoux. The sensitivity of the human Mantoux alone for diagnosing non-tuberculous mycobacterial infection was $81 \%$ for a response of $\geqslant 5 \mathrm{~mm}$ and $66 \%$ for $\geqslant 10 \mathrm{~mm}$. Ten patients had a 0 human response. Fifty five of the 59 patients had an avian response at least $2 \mathrm{~mm}$ greater than the human response.

Conclusion-The avian Mantoux is a very sensitive method of diagnosing nontuberculous mycobacterial infection in children. The human Mantoux is not sensitive enough to be used alone as a surrogate to diagnose non-tuberculous mycobacterial infection.

(Arch Dis Child 1999;80:377-379)
\end{abstract}

Keywords: non-tuberculous mycobacteria; Mantoux; lymphadenopathy

Department of Immunology and Infectious Diseases, Royal Alexandra

Hospital for Children, Westmead, NSW 2145, Australia

A J Daley

$\mathrm{D}$ Isaacs

Correspondence to: Dr A J Daley, Centre for Infectious Diseases and Microbiology Laboratory

Services, Institute of Clinical Pathology and Medical Research, Westmead

Hospital, Westmead, NSW

2145, Australia.

email: andrewd@icpmr.

wsahs.nsw.gov.au

Accepted 28 October 1998 degree. $^{2}$ A rapid and simple method for the diagnosis of non-tuberculous mycobacterial infection would have considerable clinical value because the management of the conditions varies according to the diagnosis. Furthermore, in certain situations, such as with lesions in the parotid area, excisional biopsy may lead to morbidity from facial nerve damage. We undertook this retrospective review of culture proven non-tuberculous mycobacterial infections to determine the sensitivity of skin testing with both avian sensitin and human tuberculin in children.

\section{Methods}

A retrospective review was performed of all patients with culture proven non-tuberculous mycobacterial lymph node infections who also had differential avian and human skin testing performed over a 10 year period from 1986 to 1996.

Specimens consisted of lymph node biopsies and pus swabs from various anatomical sites. All clinical isolates were forwarded to a reference laboratory for identification. From 1992 the species of non-tuberculous mycobacterium was available; before this the isolates were classified only into the Mycobacterium avium-intracellulare-scrofulaceum

(MAIS) group.

Avian and human tuberculin purified protein derivative (PPD) was obtained from the Commonwealth Serum Laboratories Limited (CSL, Parkville, Melbourne, Australia). The human tuberculin PPD was manufactured from killed $M$ tuberculosis and the avian PPD from killed $M$ avium. Both were prepared as a solution of the protein derivative in phosphate buffer with $0.5 \% \mathrm{wt} / \mathrm{vol}$ phenol as a preservative and $0.005 \%$ vol $/ \mathrm{vol}$ Tween 80 as a stabiliser. These preparations were tested for potency in sensitised guinea pigs and standardised against the World Health Organisation's first international standard tuberculins. The current tuberculins supplied by CSL in Australia have been available for over 30 years and the method of manufacture has not changed over this time. No other tuberculins have been available in Australia for routine testing during this period (CSL, personal communication, 1998).

The avian and human tuberculins were used at a strength of $100 \mathrm{IU}(0.002 \mathrm{mg}) / \mathrm{ml}$ with a dose of $10 \mathrm{IU}(0.1 \mathrm{ml}$ or $0.0002 \mathrm{mg})$ administered intradermally via a $26 \mathrm{G}$ needle using a $1 \mathrm{ml}$ tuberculin syringe into the ventral surface of the upper part of each forearm (by convention the avian in the right forearm and the human in the left forearm). The degree of 


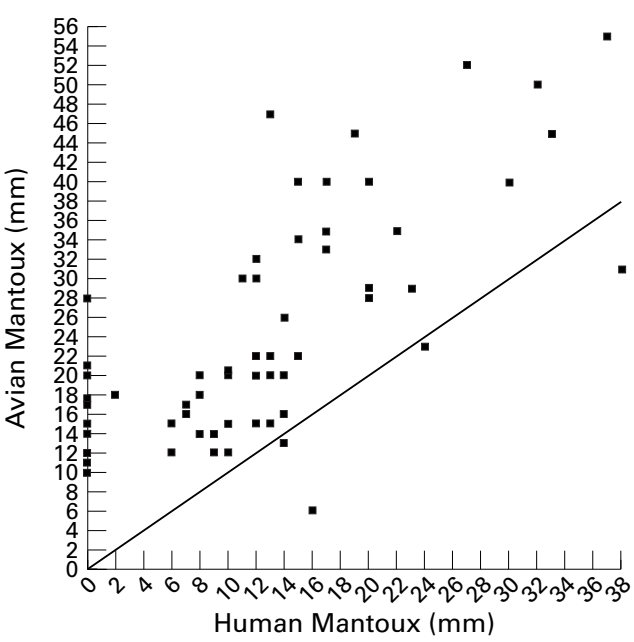

Figure 1 Mantoux responses in non-tuberculous mycobacterial infection (line of identity drawn).

induration was measured at 72 hours by a trained member of staff and recorded. No patient had significant vesiculation or ulceration at the test site.

The interpretation of both the avian and human results was based on the National Tuberculosis Advisory Council (Canberra) recommendations $^{3}$ as follows: negative, $<5 \mathrm{~mm}$ diameter; weak positive, 5-9 $\mathrm{mm}$ diameter; intermediate positive, $10-14 \mathrm{~mm}$; and strong positive, $\geqslant 15 \mathrm{~mm}$ diameter with or without vesiculation.

\section{Results}

A total of 124 patients had non-tuberculous mycobacteria isolated from lymph nodes over the 10 year study period. Differential human and avian skin testing was performed in 59 of these 124 patients.

During the six year period from 1986 to 1991 there were 16 MAIS isolates, not further speciated. From 1992 to 1996 there were $26 M$ avium isolates, $16 \mathrm{M}$ intracellulare isolates, and one $M$ scrofulaceum isolate.

The specimens were from various sites, predominantly from around the head and neck. The site of infection was available for 42 of the isolates, with 21 being from cervical abscesses (no further anatomical details supplied), seven from submandibular glands, three from parotid glands, two from the mastoid region, two from preauricular nodes, one from an occipital node, one from an axillary node, one from an inguinal node abscess, and four from various face and cheek cysts.

In our study, no patient had both a negative human and avian Mantoux result. Fifty eight of the 59 patients had an avian response $\geqslant 10 \mathrm{~mm}$, giving a sensitivity for this test alone of $98 \%$. One patient had an avian response of $6 \mathrm{~mm}$ and a human Mantoux of $16 \mathrm{~mm}$. The sensitivity of the human Mantoux alone for diagnosing non-tuberculous mycobacterial infection was $81 \%$ for a response of $\geqslant 5 \mathrm{~mm}$ and $66 \%$ for $\geqslant 10 \mathrm{~mm}$. Ten of 59 patients had a zero $(0 \mathrm{~mm})$ human response. Comparing the differential results, 55 of the 59 patients had an avian response greater than the human by at least $2 \mathrm{~mm}$ and in 52 the response was greater by at least $3 \mathrm{~mm}$ (fig 1 ).

\section{Discussion}

The diagnosis of non-tuberculous mycobacterial infection is based on a high level of clinical suspicion, appropriate delayed-type hypersensitivity skin testing, and, ultimately, excisional biopsy of the nodes with histological and microbiological confirmation. Fine needle aspiration has also been used for diagnosis with some success. ${ }^{4}$

Skin testing with PPD from $M$ tuberculosis at a strength of $5 \mathrm{IU}$ (international tuberculin units) commonly produces a positive reaction of between 5 and $10 \mathrm{~mm}$ in children with nontuberculous mycobacterial infection. This is a result of cross reaction with antigens present on the non-tuberculous mycobacteria. In areas where the prevalence of tuberculosis is low, this crossreaction has been used as a surrogate test for non-tuberculous mycobacterial infection but may in fact lead to diagnostic confusion.

Specific non-tuberculous mycobacterial sensitins have been produced but there have been problems with standardisation of the various preparations used. ${ }^{5}$ Furthermore, the definition of what constitutes a significant avian reaction has varied in the studies performedfrom any reaction equal to or greater than the corresponding reaction to tuberculin ${ }^{7}$ to that exceeding the human tuberculin reaction by 2 to $4 \mathrm{~mm}^{8}$

Several studies have attempted to assess the sensitivity of the avian Mantoux but these also included patients in whom the diagnosis was not proven on culture. Wright has recently published his Australian experience. ${ }^{9}$ All but one of his 67 patients had an avian Mantoux performed but only 29 of his patients had culture proven non-tuberculous infection, the other diagnoses being based on "diagnostic histology". A total of 63 of 66 patients had an avian Mantoux $>10 \mathrm{~mm}$, giving a sensitivity of $95 \%$ for the avian Mantoux. There were three false positives, with the final diagnoses being cat scratch disease, pilomatrixoma, and "nonspecific lymphadenopathy". Only 14 of 56 patients had a positive human Mantoux of $>10 \mathrm{~mm}$ to $10 \mathrm{IU}$ human PPD.

We were unable to estimate the specificity of the avian Mantoux because we did not have a sufficient number of children with other proven infections who had differential avian and human Mantoux testing performed. In any study it is difficult to exclude non-tuberculous mycobacterial infection completely, even if another diagnosis is made.

Different preparations of avian and human PPD are used and at different strengths (5 IU in North America, 10 IU in Australia and the UK). There is no evidence as to whether these differences alter the reliability of the Mantoux test. Our results on the sensitivity of the avian Mantoux were similar to those obtained by Margileth in $1983 .{ }^{10}$ As part of a larger study on the use of non-tuberculous mycobacteria for skin testing, he investigated 26 patients with culture proven MAI infection, 23 of whom had a reaction to the non-tuberculous sensitin at 
least $3 \mathrm{~mm}$ greater than the corresponding human PPD reaction. He also found that a fifth of these patients had a negative reaction to human PPD. In a small study of five children with culture proven non-tuberculous mycobacterial infection, Del Beccaro found that all children had a response of $>15 \mathrm{~mm}$ to the $M$ avium complex antigen used. ${ }^{11}$

Because of the small number of children with proven $M$ tuberculosis infection seen at this hospital, we were unable to compare their reaction with both the human and avian antigens. Margileth was able to do this for 22 patients and found that all had a positive human Mantoux of $\geqslant 15 \mathrm{~mm}$ and none had an avian result greater than the human one. ${ }^{10}$

The best sensitivity in our study was obtained using an avian cut off point of $\geqslant 10 \mathrm{~mm}$ and having the avian result equal to or greater than the human result. If we used a definition of a positive result being when the avian response was greater than the human response by $2 \mathrm{~mm}$ or more, the sensitivity was $93 \%$; if we used a definition of the avian response being at least $3 \mathrm{~mm}$ greater the sensitivity was $88 \%$. Using a cut off of $\geqslant 15 \mathrm{~mm}$ for the avian Mantoux may increase the specificity but decreased the sensitivity to $81 \%$ in our study.

Our study demonstrates that in countries with a low incidence of tuberculosis, the avian Mantoux is a very sensitive method for confirming the clinical suspicion of non- tuberculous mycobacteria as a cause of childhood lymphadenopathy. The use of the human Mantoux alone as a diagnostic tool is much less sensitive in this situation and potentially confusing. In areas where tuberculosis is prevalent, the differential human and avian Mantoux may be a useful aid to diagnosis.

1 Runyon EH. Anonymous mycobacteria in pulmonary disease. Med Clin North Am 1959;43:273-90.

2 Bodenstein L, Altman RP. Cervical lymphadenitis in infants and children. Semin Pediatr Surg 1994;3:134-41.

3 Commonwealth Department of Health, Australia. The Commononwealth Department of Health, Australia. The tuberculin test: review and recommendations by the National Tuberculosis Advisory Council. Canberra:
ernment Printing Service (AGPS), 1979.

4 Cox HJ, Brightwell AP, Riordan T. Non-tuberculous mycobacterial infections presenting as salivary gland masses in children: investigation and conservative management. $\mathcal{F}$ Laryngol Otol 1995;109:525-30.

5 Margileth AM. Editorial response: what is the diagnostic value of dual skin testing with tuberculous and nontuberculous mycobacterial antigens? Clin Infect Dis 1994;19:213.

6 Chaparas SD. Editorial response: dual skin testing with Mycobacterium avium sensitin and purified protein derivative in patients with $M$, avium complex infection or tuberculosis. Clin Infect Dis 1994;19:24-5.

7 Jeanes CWL, Davies JW, McKinnon NE. Sensitivity to atypical acid fast mycobacteria in Canada. Can Med Assoc $\mathcal{F}$ 1969;100:888-95.

8 Fordham von Reyn C, Green PA, McCormick D, et al. Dual skin testing with Mycobacterium avium sensitin and purified protein derivative: an open study of patients with M. avian complex infection or tuberculosis. Clin Infect Dis 1994;19:15-20.

9 Wright JE. Non-tuberculous mycobacterial lymphadenitis. Aust N Z F Surg 1996;66:225-8.

10 Margileth AM. The use of purified protein derivative mycobacterial skin test antigens in children and adolescents: purified protein derivative skin test results correlated with mycobacterial isolates. Pediatr Infect Dis 1983;2:225-31.

11 Del Beccaro MA, Mendelman PM, Nolan C. Diagnostic usefulness of mycobacterial skin test antigens in childhood lymphadenitis. Pediatr Infect Dis f 1989;8:206-10. 